\title{
ENNEMIES MALGRÉ NOUS, SCEURS MALGRÉ TOUT : LE THÈME DU « TÉMOIGNAGE » DANS ÉTOILE ERRANTE DE J. M. G. LE CLÉZIO
}

\author{
Nesrine EL-ZINE \\ Université de Sana'a, Yémen
}

\begin{abstract}
En): Jean-Marie Gustave Le Clézio was awarded the Nobel Prize for Literature in 2008 with a literary production marked by a mixture of several traits of different literary movements in the 20th century. In his work Étoile Errante (Wandering Star), Le Clézio sheds light on a double human drama marked by exodus, suffering, and the uncertainty of fate. While reflecting the true complexity of the human's dimension in this conflict, the story of Nejma and Esther proves to us that war, fueled by hatred and intolerance towards others, brings the two heroines together more than it divides them; it unites them by a kind of equality of misfortune and suffering. In this study, we will touch the importance of writers' role as spokespersons in human society. However, the main objective will be to present the theme of the "Témoignage" (Testimony) in Étoile Errante (Wandering Star) and to try to analyze its power from these two points of view: On the one hand, the power of testimony to denounce the outraged zeal and the unjustifiable attachments (religious, political, ideological, ethnic, etc.), which can generate tragedies in human societies. On the other hand, the power of testimony to make the voices of these tragedies' victims, who are often oppressed because of political, religious, ideological intolerance, heard.
\end{abstract}

Keywords (En): literary engagement; testimony; intolerance; double human drama

Mots-clés (Fr) : engagement littéraire ; témoignage ; intolérance ; double drame humain

\section{Introduction}

Le phénomène du «Témoignage » est assez répandu dans la littérature du vingtième siècle, dans laquelle il joue le rôle très important de refléter une certaine réalité historique ou sociale que le lecteur n'a pas forcement vécue. Felman et Laub nous le précisent en analysant le témoignage comme l'un des aspects les plus essentiels dans le roman La Peste de Camus. Ils expliquent que l'auteur/le narrateur, une fois reconnu comme témoin fidèle d'un certain événement historique, devient capable de lier (comme un pont, selon leur propre expression) le domaine de la narration dans ses œuvres littéraires aux différents événements/faits historiques dont il a témoigné tout au long de sa vie.

Joining events to language, the narrator-as-eyewitness is the testimonial Bridge which, mediating between narrative and history, guarantees their Correspondence and adherence to each other. This bridging between narrative and history is possible since the narrator is both an informed and an honest witness (FELMAN \& LAUB, 1992 : 24).

A cet égard, il est crucial de souligner l'importance que les écrivains accordent aux témoins et à leurs témoignages. En effet, ces derniers deviennent le miroir des faits et des événements vécus, ils nous permettent de visionner leur drame par leurs propres yeux, selon Claude Burgelin : 
Le témoin se soumet à l'exigence d'une vérité, ou tout du moins d'une exactitude, qu'il tente de transmettre, mais qu'il ne saurait altérer ou adultérer. Une instance transcendante, la vérité, qui ne se confond point avec l'exactitude, mais qui ne transige point avec elle, régit le dire du narrateur, le soumet à sa loi. (BURGELIN, 1995 : 79-80)

Dans cette même ligne de pensée et pour mieux comprendre l'importance du thème du «Témoignag » dans l'œuvre leclézienne, rappelons-nous d'une citation de l'auteur, extraite de son discours de réception du prix Nobel le 6 décembre 2008 à Stockholm, et dans lequel il expliquait la raison pour laquelle un écrivain voudrait écrire en affirmant que : «L'écrivain, depuis quelque temps déjà, n'a plus l'outrecuidance de croire qu'il va changer le monde, qu'il va accoucher par ses nouvelles et ses romans un modèle de vie meilleure. Plus simplement, il se veut témoin. » (LE CLÉZIO, 2008)

Ainsi, l'objectif principal dans cette étude est de souligner le thème du « Témoignage » dans le roman Étoile Errante. Nous ambitionnons d'analyser son rôle et son importance dans :

1. La dénonciation des zèles outrés et des attachements injustifiables qui peuvent engendrer des drames dans les sociétés humaines.

2. La libération de la parole des victimes, ceux qui sont souvent opprimés ou bien « capturés » (pour emprunter la terminologie utilisée par Le Clézio) par l'intolérance politique, religieuse voire idéologique.

\section{Engagement littéraire et société}

Depuis toujours, le rôle joué par les écrivains dans la société est incontestable. Ils nous transmettent dans leurs écrits, des drames sociaux et humains, des crises économiques et historiques, ou encore des tensions politiques et idéologiques, etc... Ils sont, grâce à leurs ouvrages littéraires, les porte-paroles non seulement de leur société, mais de toute l'humanité. De ce point de vue, rappelons que c'est à partir de la fin du $\mathrm{XIX}^{\mathrm{e}}$ siècle et surtout au $\mathrm{XX}^{\mathrm{e}}$ siècle que la littérature française dite engagée a fait son apparition officielle. Une approche militante régissait l'esprit et les idées des écrivains de cette époque, notamment Gide qui dénonçait les abus du système colonial dans son journal Voyage au Congo publié en 1927. Jean-Paul Sartre, quant à lui, définit un écrivain dit engagé de la façon suivante « je dirai qu'un écrivain est engagé lorsqu'il tâche à prendre la conscience la plus lucide et la plus entière d'être embarqué, c'est-à-dire lorsqu'il fait passer pour lui et pour les autres l'engagement de la spontanéité immédiate au réfléchi » (Sartre, 1948 : 98-99). Il fait le lien entre le « silence » de Flaubert et de Goncourt face à la Commune, il explique que : «L'écrivain est en situation dans son époque: chaque parole a des retentissements. Chaque silence aussi. Je tiens Flaubert et Goncourt pour responsables de la répression qui suivit la Commune parce qu'ils n'ont pas écrit une ligne pour l'empêcher. » (FLOREY, $2013: 27$ )

Néanmoins, bien que la littérature dite engagée ait atteint son apogée et son intensité au $\mathrm{XX}^{\mathrm{e}}$ siècle suite à la Seconde Guerre mondiale, il est indéniable que l'engagement des écrivains était déjà présent bien avant cette époque-là. En effet, dès le $\mathrm{XVI}^{\mathrm{e}}$ siècle, les écrivains français ont assumé la responsabilité de démontrer 
les maux de leur société et d'en dénoncer ses vices. Ainsi, Montaigne luttait dans ses œuvres contre l'intolérance. De même, le poète de la Pléiade, Ronsard a publié pendant la première Guerre de Religion (1562-1563), les Discours des misères de ce temps dans lesquels il attaquait les chefs spirituels protestants et condamnait leur rébellion ayant provoqué les Guerre de Religion. Ensuite, viennent au XVII ${ }^{\mathrm{e}}$ siècle les écrivains "satiristes", qui ont aussi pris position envers les défauts qui apparaissaient dans la société française de l'époque, citons à titre d'exemple Molière qui dénonçait l'avarice dans L'Avare, l'hypocrisie religieuse et les faux dévots dans Tartuffe et luttait pour que les femmes obtiennent le droit à l'éducation dans L'École des femmes. Au XVIII ${ }^{\mathrm{e}}$ siècle, les philosophes des Lumières ont aussi mis en avant les maux de leurs sociétés, par exemple, Voltaire qui a remarquablement combattu l'obscurantisme et l'intolérance dans des ouvrages tels que les Lettres philosophiques, Zadig ou Candide.

Quoi qu'il en soit, le thème du drame de la guerre est l'un des plus provocants pour les écrivains engagés, « la guerre porte cette tension extrême pour l'écrivain : la nécessité d'écrire tandis qu'on ne peut plus parler» (YANA, 1986: 4). Ainsi, n'est-il pas surprenant de voir les écrivains, de toutes les époques, se manifester contre les guerres et les horreurs qu'elles engendrent dans les sociétés.

\section{Réflexion sur le roman Étoile Errante}

\subsection{Un canevas multidimensionnel}

Il est certain que la reconnaissance de la profondeur du drame humain devrait être le thème le plus important dans toutes les négociations entre les deux parties de l'interminable conflit israélo-palestinien. En effet, le sort des réfugiés palestiniens est un thème chargé en émotion. Il touche de très près au sentiment national qui s'est forgé pendant les nombreuses années d'exil du peuple palestinien. D'ailleurs, ce drame de l'exil des Palestiniens est connu de tous depuis le 29 novembre 1947, date qui marque les premières initiatives pour l'adoption du plan de partage de la Palestine par l'ONU (résolution 181). Cette résolution a tracé le chemin des conséquences d'un des conflits les plus persistants de l'histoire de l'humanité en général, et de celle du Moyen-Orient en particulier, avec en premier lieu, la guerre entre Arabes et Juifs dès les premiers jours qui ont suivi l'adoption par l'ONU du plan de partage, et ensuite avec l'attaque de l'État d'Israël par les armées arabes au lendemain de sa déclaration d'indépendance le 14 mai 1948 (ISRAELI, 2002 : 121).

Dans son roman Étoile Errante (1992), J. M. G. Le Clézio met en évidence le traitement violemment infligé au peuple palestinien, et dont la population souffre encore de nos jours. De même, le roman évoque la persécution des Juifs par les nazis pendant la Deuxième Guerre mondiale. La trame dans ce roman n'est point celle de Nejma, ni d'Esther; ce n'est même pas le récit de deux jeunes filles qui ne se rencontrent qu'une seule fois pour ne plus jamais se revoir, ce n'est ni l'un ni l'autre. C'est plutôt l'histoire de deux peuples, de générations qui se succèdent, de deux communautés qui, à un certain moment, doivent coexister et cohabiter. Nous ne saurons jamais définir le début de cette histoire ni sa fin. D'ailleurs, celle-ci rassemble maints sentiments bouleversants qui reflètent de près la déchirure 
résultant sur l'un des problèmes les plus pénibles et les plus persistants dans la région du Moyen-Orient.

Des milliers de victimes sont à dénombrer des deux côtés, et nous soulignons avec beaucoup d'insistance le mot « victimes », à l'instar des deux héroïnes-témoins d'Étoile Errante, Nejma et Esther. Rien ne les opposait et leurs chemins n'auraient probablement jamais dû se croiser, ou alors dans d'autres circonstances plus favorables et amicales. À un certain moment de leur histoire, le destin s'est imposé en permettant la rencontre des deux jeunes filles, pourtant, ce même moment représente aussi celui de leur séparation à jamais. Les témoignages de leur vie pendant la période d'exode et de fuite réalisés par les deux héroïnes sont bien significatifs. Ils nous transmettent beaucoup de leur souffrance personnelle et celle de leurs communautés.

Cependant, peut-on suggérer que Le Clézio ait écrit Étoile Errante en ayant à l'esprit de traiter une identité ou une idéologie politique ? Il est fort probable que ceci ne soit pas le cas, surtout si on considère que le roman a été écrit dans les années 1980, ce qui coïncidait avec l'Intifada du peuple palestinien en 1982. Pourtant, Le Clézio ne publie son roman qu'en 1992 comme pour éviter toute connotation politique qui aurait pu être suggérée dans le roman à cette époque-là. Plutôt dans l'optique de lutter pour le droit des enfants à vivre en liberté et en paix, Le Clézio dédie son roman "aux enfants capturés ». Aussi, grâce à un humanisme très minutieux pour transcrire la réalité du malheur des victimes du conflit israélopalestinien, le roman a reçu le grand prix de la Ligue internationale des Droits de l'Homme à sa publication en 1992.

Certains voient dans le conflit israélo-palestinien l'évident résultat d'une longue histoire d'oppression, de persécution et d'injustice. Dans cette même ligne de pensée, Anne Millat citée par Bruno Thibault décrit le conflit présenté dans Étoile Errante (EE) comme « une histoire en miroir où les persécutés deviennent à leur tour des persécuteurs. » (THIBAULT, 2009a : 165). En effet, le peuple juif qui avait longtemps subi la persécution et l'injustice en Europe s'est révolté, mais sa révolution s'est traduite par l'agression des droits d'un autre peuple, à savoir le peuple palestinien. Comme le confirme Rymond Mbassi Atéba pour qui, il est impossible de : «[...] nier le fait que le peuple juif a connu depuis la lointaine antiquité, en passant par le Moyen Âge où l'antisémitisme devient réel, une histoire tourmentée par la captivité et l'agressivité de l'altérité proche orientale ou occidentale. » (MBASSI ATEBA, 2008 : 292).

Cependant, la révolte des Juifs s'est déclarée contre le peuple palestinien qui, quant à lui, n'était d'aucune façon mêlé au désarroi ou à la souffrance des premiers. Ainsi, le peuple palestinien est devenu, à son tour, la victime d'une injustifiable oppression exercée par le peuple juif, qui avait été la victime opprimée auparavant. Autrement dit, le persécuté devient le persécuteur d'une autre victime plus faible. Selon Tahar Ben Jelloun, le témoignage sur l'injustice juive envers le peuple palestinien que Nejma nous offre dans Étoile Errante révèle :

Mieux que n'importe quelle analyse socio-historique de la question palestinienne, le texte de J.M.G. Le Clézio rend compte avec beaucoup de sobriété et de justesse de ce que fut l'expulsion des Palestiniens de leurs terres en 1948. C'est la voix de Nejma, arrivée à seize ans au camp de réfugiés qu'on entend tout au long de ce récit bouleversant [...]. Nejma, qui raconte simplement 
la mémoire des jours vécus dans le camp, verra la mort faire lentement son travail... Elle dit qu'elle a honte, honte de ce que les hommes sont capables de provoquer comme détresse. (BEN JELLOUN, $1988: 31)$

De ce fait, on peut interpréter que l'exclusion et l'oppression voire l'intolérance envers l'Autre pourrait être une des raisons du conflit israélo- palestinien. Le roman nous montre d'un point de vue humaniste les conséquences de cette intolérance et comment cela va terriblement affecter la vie des civils impuissants en proie à la terreur et à l'exode forcé. Par ailleurs, bien que le roman présente un « double» drame humain dont les faits misérables sont partagés par les deux nations opposées, Le Clézio ne décrit pas de scènes cruelles ou terrifiantes de la guerre. Ce sont toujours des éléments rapportés par écho et par ce que les autres en racontent :

Les nouvelles disaient que les fermiers juifs avaient été assassinés, dans la colonie d'Ataroth... Les gens disaient que, maintenant que les Anglais étaient partis, tout allait s'arranger. D'autres disaient que cette guerre ne faisait que commencer, que ce serait la troisième guerre mondiale. (EE : 216-217)

Pourtant, on saisit la souffrance des deux protagonistes et de leurs communautés et on éprouve une profonde compassion à leur sort. Le roman semble nous transmettre une sensation de découverte des êtres humains qui vivent et subissent les conséquences de cette guerre. Ainsi, serait-il peut-être légitime de penser que la guerre n'est pas, en elle-même, ce que Le Clézio voulait partager ? Certes, nous accordons que la guerre n'est pas son objectif principal dans ce livre, mais qu'il y vise plutôt les effets de cet événement sur la vie des civils. Par contre, il est indéniable que les traits stoïciens et l'influence bouddhiste sont bien reflétés chez Le Clézio. En effet, les conséquences de la guerre et le double drame humain autour duquel Étoile Errante gravite sont marquées par beaucoup de détails de « contemplation » voire de méditation. Ceux-ci sont présentés à travers la réflexion et l'observation attentive faites par les deux personnages-héroïnes. Cependant, cette contemplation-méditation transpose la mémoire des expériences de douleur à un « ressentir » cosmique des moments inoubliables vécus et à l'acceptation des faits irréguliers de la vie. C'est grâce à cette pratique, telle qu'elle est revendiquée dans toute l'œuvre leclézienne, que l'on peut se libérer des maux de la vie civilisée.

Autrement dit, on s'aperçoit que le mal peut aller de pair avec le bien et on accepte cette cohabitation avec conviction «je crois qu'avec le seul plaisir de décrire la lumière ou la mer, ou même les villes [...] on peut atteindre une certaine liberté » (Lire, 2008 : 52). Esther aussi nous le confirme, elle nous apprend qu'elle a réussi à oublier " les villes noires, les trains, la peur, tout est reste derrière nous »" (EE : 164), elle se sent libérée grâce à la contemplation de la nature autour d'elle, « J'ouvre les yeux, la mer et la lumière me brûlent jusqu'au fond de mon corps, mais j'aime cela. Je respire, je suis libre. Déjà je suis portée par le vent, par les vagues. Le voyage a commencé » (idem). 


\subsection{Un drame à double façade}

Il est important de comprendre le rapport mystérieux qui relie étroitement les deux jeunes filles du roman. En effet, il existe un rapprochement indirect entre les deux protagonistes. Comme le décrit Thibault :

La rencontre de Nejma et d'Esther montre que le rapport à l'Être et le rapport à l'Autre sont indissociables. L'exécration de l'Autre n'autorise que la vaine promotion de soi. En rejetant l'Autre, c'est la " projection » qui opère et qui domine la relation. Or, dans les deux «cahiers noirs » où elles notent leurs réflexions et rédigent leurs journaux intimes, Esther et Nejma cherchent au contraire à garder en mémoire le visage de l'Autre et à y inscrire l'espace du « transfert. » (THIBAULT, 2009b: 104).

Ainsi, un rapport enchevêtré semble régner dans les récits d'Esther et de Nejma. Leurs récits se renvoient l'un à l'autre implicitement. D'ailleurs, bien que les deux jeunes filles appartiennent à deux communautés opposées par leur intérêt politique, elles partagent plusieurs points communs et on peut même dire que leurs destins se ressemblent. Cela commence déjà avec leur prénom : Esther et Nejma. Ils ont la même signification «Étoile » dans deux langues différentes. Toutes les deux ont été forcées de quitter leur lieu d'origine à cause de la guerre. Elles passent leur vie à la recherche d'un endroit "sûr » où la paix règne et où elles peuvent se sentir en sécurité et vivre sans crainte. Malheureusement, leur errance pour rechercher cet endroit utopique ne semble pas s'achever :

Soudain, de la troupe se détacha une très jeune fille. Elle marchait vers Esther. Son visage était pâle et fatigué, sa robe pleine de poussière [...] La jeune fille s'approcha d'elle jusqu'à la toucher. Ses yeux brillaient d'une lueur étrange, mais elle ne parlait pas, elle resta immobile avec sa main posée sur le bras d'Esther, comme si elle allait dire quelque chose. Puis, de la poche de sa veste elle sortit un cahier vierge [...] elle écrivait son nom, comme ceci, en lettres majuscules : NEJMA. Elle tendit le cahier et le crayon à Esther, pour qu'elle marque aussi son nom. Elle resta un instant encore, le cahier noir serré contre sa poitrine, comme si c'était la chose la plus importante du monde. Enfin, sans dire un mot, elle retourna vers le groupe des réfugiés qui s'éloignait. ( $E E: 219)$

Regardons de près ce passage clé du roman. Il nous présente la rencontre rapide et inattendue entre Esther et Nejma au village de Latrun près de Jérusalem. D'abord, on peut parfaitement sentir la tension émotionnelle accompagnant cette brève rencontre qui crée une sorte d'alliance mystérieuse entre les deux jeunes filles, et qu'Esther va garder gravée en elle tout au long de sa vie errante. "J'ai rêvé de ce cahier. Je le voyais dans la nuit [...]. J'ai rêvé que j'avais lu ce qu'elle racontait, pour moi seule, une histoire d'amour et d'errance qui aurait pu être la mienne » $(E E: 315)$. En effet, l'impact de leur rencontre a été très fort. Elles n'ont échangé qu'un regard et leurs noms, mais après cet événement, elles ont souvent pensé l'une à l'autre. Esther dit que Nejma est sa sœur perdue. À un certain point de son récit, Esther raconte qu'elle a acheté un cahier noir semblable à celui que Nejma avait lors de leur rencontre, et qu'elle y a écrit le nom de Nejma sur la première page. En fait, elle relate sa vie personnelle dans ce cahier comme pour mettre en exergue les aspects similaires de leurs existences. Comme nous pouvons le comprendre, Esther est complètement hantée par Nejma, mais en même temps, elle reconnaît la 
différence de leur destin. En effet, si Esther est à la recherche de Nejma, c'est pour mettre fin à son errance, mais cette dernière restera toujours une étoile errante parce que son destin ne pourra jamais se définir par un endroit ou un moment spécifique.

Notons aussi que, dans ce même passage de leur unique rencontre, Nejma est la première à faire un pas vers Esther. C'est elle qui prend l'initiative d'ouvrir ce dialogue silencieux. En tout cas, son initiative n'est qu'une façon d'affirmer son identité, comme le note K. Levy : «It's important to note that Nejma's action is neither a request nor a plea, but rather an affirmation of her own dignity and identity and an offer that Esther share her own » (LEVY, 1998 : 48).

Par ailleurs, il est capital de noter la différence qui apparaît dans le comportement d'Esther envers les réfugiés arabes par rapport aux réactions des autres femmes juives. En effet, lorsqu'elles arrivent près du camp de Nour Chams, celles-ci notent avec beaucoup de haine que ces femmes réfugiées méritent tout ce qui leur arrive, en confirmant que : " Il n'y a pas d'innocents, ce sont les mères et les femmes de ceux qui nous tuent» $(E E: 220)$. Or, le récit d'Esther semble porter un sentiment de responsabilité et de culpabilité envers le sort de Nejma et de ses semblables. En effet, Esther fait allusion à maintes reprises au regard interrogatif de Nejma, et plus encore, au rapport étroit qui fait que cette dernière vient vers Esther avec l'intention de la considérer personnellement. «Elle venait de l'exil, des pays de sécheresse et de l'oubli, seule, pour me considérer » (EE:316).

De même, l'influence que Nejma exerce sur Esther a un rôle très significatif dans la vie de celle-ci. C'est l'image de Nejma, pleine de la souffrance et de l'humiliation subies par les réfugiés, qui est restée gravée dans la mémoire d'Esther pendant son séjour à Montréal. Plus tard, cette même image pousse Esther à repartir pour Jérusalem afin d'aider et d'apaiser la souffrance de ces gens persécutés, peutêtre parce qu'elle espérait aider les nouvelles générations, comme celle de son fils, à envisager une nouvelle perception du conflit et à ouvrir le chemin d'une coexistence éventuelle entre les deux peuples, comme le précise K. Levy : « Nejma likewise influences Esther's decision to return to Israel. The image of a solitary and still wandering Nejma haunts Esther during her student years in Montreal but helps her recognize her responsibility to alleviate some of the suffering in Israel. » (LEVY, 1998 : 49)

Par ailleurs, tout au long de son errance, Esther admettait son besoin de comprendre le mal auquel elle avait réussi à échapper au cours de sa vie. C'est probablement pour cette raison qu'elle voulait absolument retrouver Nejma : «Je ne savais pas ce que je cherchais, ce que je voulais voir. C'était comme une plaie au cœur, je voulais voir le mal [...]. Si je ne trouve pas où est le mal, j'aurai perdu ma vie et ma vérité. Je continuerai à être errante » $(E E: 335)$.

Les ressemblances entre l'histoire des deux héroïnes du roman sont incontestables. Étant toutes les deux victimes de la différence idéologique qui mène aux conflits politiques et historiques, leurs vies reflètent une série de départs incessants, une fuite voire une errance. Pour Esther, tout commence dans les Alpes du Sud pendant l'été 1943 dans un petit village de l'arrière-pays niçois où sont cantonnées des familles juives surveillées par les Italiens et les nazis. Dans ce village habite Hélène Grève, une adolescente juive. Hélène est un surnom, car le prénom « Esther » étant un prénom juif, il serait dangereux pour elle de le garder. Elle doit 
quitter son village, Saint-Martin de Vésubie, avec sa mère après la perte de son père. À la fin de la guerre, son errance continue; en compagnie de sa mère et d'autres familles juives, elle quitte l'Italie et prend le chemin mystérieux et incertain vers Jérusalem, ladite Terre Promise des Juifs. Enfin, elle retourne en France, à SaintMartin, pour enterrer sa mère. D'ailleurs, Esther au début de son témoignage, nous fait bien ressentir l'amertume qu'elle a éprouvée au moment de quitter SaintMartin :

C'était la première fois, c'était une douleur, Esther s'apercevait qu'elle n'était pas comme les gens du village. Eux, pouvaient rester chez eux, dans leurs maisons, ... elle devait marcher avec ceux qui, comme elle, n'avaient plus de maison, n'avaient plus droit au même ciel, à la même eau. Sa gorge se serrait de colère et d'inquiétude, son cœur battait trop fort dans sa poitrine. ( $E E$ : 92).

\section{Pourquoi Témoigner?}

\subsection{Témoigner pour libérer sa voix}

Le Clézio donne aux deux narratrices, Nejma et Esther, l'opportunité de s'exprimer, et ce probablement parce qu'il pense que permettre à des personnes oubliées comme Esther et Nejma de le faire et de parler de soi est important : « J'ai le sentiment de l'impérieuse nécessité d'entendre d'autres voix, d'écouter des voix qu'on ne laisse pas venir jusqu'à nous, celles de gens qu'on n'entend pas parce qu'ils ont été dédaignés trop longtemps, [...] mais qui ont tellement de choses à nous apporter » (CORTANZE DE, $2009: 24$ ).

Ainsi, les deux protagonistes racontent le récit de leur expérience personnelle et peut-être aussi celle des autres. D'ailleurs, il est à souligner qu'il s'agit de la première fois où la parole est accordée à des voix féminines dans le répertoire littéraire de Le Clézio. Celui-ci semble reconnaître le rôle essentiel et indépendant joué par les femmes dans le contexte de la guerre. En effet, on insiste beaucoup dans Étoile Errante sur la participation ainsi que sur la présence active des femmes dans ce drame humain, que ce soit du côté des réfugiés palestiniens ou celui des juifs.

Dans leurs témoignages, chacune des deux narratrices essaie de raconter son malheur, ses défis et sa souffrance. Pourtant, les ressemblances dans leurs deux témoignages paraissent beaucoup plus nombreuses que les différences. Mais pourquoi racontent-elles leurs histoires ? Pourquoi font-elles ces témoignages ? Estce pour le plaisir? Certainement pas ! Or, le tableau que chacune d'elles nous peint ne semble offrir, dans l'ensemble, ni gaieté ni bonheur. Ainsi, il s'agit bien d'une prise de position pour partager leur drame et briser le silence qui entoure la vie de personnes telles qu'Esther et Nejma. Le Clézio leur donne la parole pour qu'elles deviennent narratrices, mais en même temps, il leur accorde le rôle de témoin des événements politiquement et historiquement réels où un double drame humain surgit avec force: d'abord, la persécution des Juifs par Hitler et son mouvement nazi présenté dès le début du récit d'Esther, et ensuite les événements marquant le conflit israélo-arabe avec, d'une part, l'établissement d'un État israélien accompagné de rafles massives de juifs voulant s'installer en Terre promise, et d'autre part, l'éparpillement du peuple palestinien chassé de ses propres terres pour être consigné dans des camps de réfugiés. 
Cependant, les témoignages d'Esther et de Nejma ne semblent pas vouloir dépeindre les événements historiques de manière spécifique, mais s'attarder plutôt sur la vie des gens qui les ont vécus; la vie des innocents qui ont souffert de la guerre. Dans ce contexte, les lieux sont réels, mais l'histoire racontée n'a pas forcément eu lieu. Pourtant, on ne peut confirmer son côté fictif ou imaginaire. De même, on ne peut dire que les événements racontés sont irréalistes puisqu'il s'agit d'un drame qui demeure actuel désormais. Il faut aussi souligner que ce phénomène de témoignages dont nous avons parlé se présente essentiellement à travers une expérience concrète voire matérielle à laquelle les témoins participent physiquement dans leur propre chair. Autrement dit, les deux jeunes filles témoignent dans leurs récits de la souffrance qu'elles ont vécue durant leur parcours vers l'inconnu. Elles nous transmettent donc leurs émotions et leurs sentiments accablés par la crainte, la poursuite, l'insécurité, la misère, et l'humiliation. Elles nous incitent donc à prendre position vis-à-vis de cette injustice qu'elles ont longuement supportée.

En effet, dans une étude faite sur l'écriture testimoniale des enfants sur l'Holocauste et le génocide, l'auteur présente l'aspect littéraire du témoignage et fait la distinction entre le témoignage des enfants et celui des adultes tel que théorisé par Aharon Appelfeld dans son œuvre L'héritage nu :

\footnotetext{
Si pour les adultes les témoignages doivent être factuels, chronologiques et fidèles, pour les enfants - que l'on a refusé de considérer comme des témoins - faute de pouvoir reconstituer le passé par la mémoire, il s'agit de recourir à la fiction, à l'expression des sensations et des sentiments, en d'autres mots à la perception, pour procéder à une « reconstruction ». (PEJOSKABOUCHEREAU, $2014:$ 1).
}

C'est exactement cette «reconstruction » que les témoignages d'Esther et Nejma nous renvoient. Elles essayent de nous faire sentir, voire revivre, l'histoire d'un malheur collectif. En effet, leurs témoignages ne se limitent pas à présenter leurs craintes et souffrances personnelles, car toutes les deux nous racontent leurs histoires comme si elles étaient les porte-paroles de toute une génération ayant vécu ces mêmes circonstances « Rejected by mankind [...] the condemned persist in surviving-not only to survive, but to testify. The victims elect to become witnesses » notent FELMAN et LAUB (1992 : 117). Autrement dit, Esther et Nejma sont des victimes elles-mêmes, mais elles choisissent de témoigner de l'injustice que vivent leurs semblables pour que leur récit soit gravé dans l'Histoire malgré tout, et parce que «d'une génération à l'autre, les hommes pouvaient oublier l'endroit où ils avaient vécu, les ressentiments qu'ils avaient eus. Ils n'avaient pas d'histoire » (EZINE, $1995: 63$ ).

\subsection{Témoigner pour dénoncer}

Toujours dans l'attente de réaliser son rêve et celui de sa mère de se retrouver en sécurité et d'atteindre le paradis promis, la jeune fille de dix-sept ans savait parfaitement qu'elle devait quitter l'Europe pour prendre le chemin vers la Terre promise, même si elle craignait de ne pas pouvoir y arriver un jour : « Je sais que je vais quitter ce pays, pour toujours. Je ne sais pas si j'arriverai là-bas, mais nous allons bientôt partir » (EE: 143). Il est clair que les sentiments de doute et d'incertitude étaient toujours présents et bien pesants dans les rêves d'espérance 
d'Esther, et probablement des autres Juifs aussi puisque, comme déjà précisé, Esther ne racontait pas que son histoire et celle de sa mère, mais l'histoire de tous les Juifs de cette période qui désiraient partir pour s'installer à Jérusalem.

Elle part donc vers l'inconnu avec sa mère et les autres. Son témoignage semble bien mettre en évidence la hantise d'un attachement inexplicable à cet inconnu, une sorte de fanatisme injustifiable. Il est curieux que l'incertitude ne découle pas du doute sur la réalité de l'existence d'une terre utopique qu'est la Terre Promise, mais plutôt sur la possibilité d'atteindre ce paradis. En effet, dans l'imaginaire de ces immigrants juifs, Jérusalem représentait la Terre du salut et de la paix, la Terre de liberté qui ne ressemblait à aucun autre endroit. Bref, il s'agissait d'une ville extraordinaire.

D'ailleurs le témoignage d'Esther est très significatif à ce propos quand elle explique : "Je pensais que ça devait être la plus belle et la plus grande ville du monde, pas comme Paris en tout cas, car il n'y avait sûrement pas là-bas des rues noires ni d'immeubles vétustes, ni de gouttières crevées ou couraient les armées de rats $[\ldots]$ Jérusalem était sûrement autre chose » $(E E: 155)$.

Un peu plus loin, elle continue avec la description illusoire qu'on lui avait faite :

Une ville comme un nuage, avec des dômes et des clochers et des minarets, des collines tout autour, plantées d'orangers et d'oliviers, une ville qui flottait au-dessus du désert comme un mirage, une ville où il n'y avait rien de banal, rien de sale, rien de dangereux. Une ville où on passait son temps à prier et à rêver $(E E: 155)$.

D'après cette description éblouissante de la ville de Jérusalem, on peut parfaitement comprendre le paradoxe et l'illusion dans lesquels les immigrants juifs vivaient en rêvant d'une nouvelle vie. Tout de même, la contradiction la plus importante à souligner est le fait que toute cette belle description ne fait aucune allusion aux habitants déjà installés et depuis bien longtemps dans cet endroit de rêve.

Ainsi, le départ vers cette terre utopique représentait pour la plupart de ces immigrants juifs une renaissance permettant de se soulager d'un passé douloureux et sombre :

J'ai l'impression que j'ai jamais cessé de marcher et de courir, depuis le jour où nous sommes descendues de la montagne à travers les hautes herbes qui coupaient mes lèvres et mes jambes, et que depuis ce jour mon cœur n'a cessé de battre trop vite et trop fort, de cogner dans ma poitrine comme un animal apeuré ( $E E: 182)$.

Donc, la terre de Jérusalem est devenue une porte symbolique qui mène vers un nouveau monde où ces personnes immigrantes ne seraient plus chassées et où elles n'auraient plus rien à craindre. « Maman avait parlé souvent de cela, elle aussi. Les derniers temps, à Paris, elle ne vivait plus que pour ce nom de Jérusalem » ( $E E$ : 155). Et aussi «A Jérusalem, disait l'oncle Simon Ruben, il n'y que les anges qui vous attendent » $(E E: 159)$. C'est bien l'image d'un vrai paradis. La légende est de plus en plus radicalisée dans l'esprit de ces immigrés. " Celui qui a tout fait est notre Père, nous sommes nés de lui. Eretz Israël, c'est l'endroit où nous sommes nés, l'endroit où la lumière a brillé pour la première fois » $(E E: 184)$. Ainsi, nous pouvons constater qu'Esther, comme les autres, s'est laissée tromper par l'illusion 
de l'existence d'une ville magique dont la paix et la sérénité sont symboliques. D'ailleurs, elle reconnait bien la fragilité trompeuse de ce rêve fallacieux « Il y a si longtemps que cela semble une légende [...] Jérusalem [...] ville lumière [...] mirage $[\ldots]$. Où est cette ville ? Existe-t-elle ?» $(E E: 342)$.

D'ailleurs, nous trouvons intéressant de noter le lien étroit qui amalgame la forte croyance à une Terre promise avec toutes les connotations religieuses présentées au long du témoignage d'Esther. En effet, celle-ci semble découvrir le domaine de la religion qui est assez inconnu et mystérieux pour elle. En fait, on apprend dès le début du récit qu'elle appartient à une famille laïque, pour qui la question de la religion était essentiellement fondée sur la liberté de choix. «Ils (les parents d'Esther) pensaient toujours que la religion était une affaire de liberté » (EE:79). Esther nous raconte sa première expérience avec la religion lors de la cérémonie de Shabbat où elle s'est trouvée poussée par une force inconnue pour entrer dans le chalet et assister aux rites religieux qui y étaient accomplis. Esther décrit la cérémonie avec beaucoup d'émotion. «C'était pareil à un vertige » nous précise-telle. Tout lui semblait impressionnant : les flammes, les voix alternées des chanteurs avec celles des chanteuses, leur langage "étrange », le balancement rythmé des corps et même l'odeur suintante des bougies. Ici, il est important de signaler l'impact profond que laissera cette expérience religieuse dans l'existence d'Esther. Cette dernière insiste sur le fait qu'au cours de cette cérémonie, elle a ressenti la certitude que rien ne pouvait menacer leur vie, et que rien d'autre ne pouvait avoir d'importance. Elle nous dit qu'à ce moment-là, elle «pouvait franchir le temps et les montagnes, comme l'oiseau noir que lui montrait son père, jusque de l'autre côté des mers, là où naissait la lumière, jusqu'à Eretz Israël » (EE : 82).

Ainsi, on voit Esther établir un rapport solide entre la prière et le rêve d'arriver à la terre promise dans la phrase précédente. Autrement dit, la prière prend un aspect presque magique pour la réalisation de ce rêve éternel. Lors de son passage sur le bateau Sette Fratelli, Esther nous fait remarquer l'effet profond que la prière exerçait sur les autres passagers de ce même bateau, qui :

Regardent la mer, leurs lèvres bougent aussi, comme s'ils priaient. Pour la première fois de ma vie, je suis en train de prier, moi aussi. C'est en moi, je le sens, au fond de moi, malgré moi. C'est dans mes yeux, c'est dans mon cœur, comme si j'étais en dehors de moi et que je voyais audelà de l'horizon, au-delà de la mer. Et tout ce que je vois maintenant signifie quelque chose, m'emporte, me lance dans le vent, au-delà de la mer $(E E: 167)$.

D'autre part et au cours de son récit, Esther nous parle de Jacques le Berger et de ses conversations avec Reb Joël qui racontait l'histoire de Jérusalem et celle du peuple juif. Elle aimait les écouter et nous rappelle ses impressions en décrivant que : « Le Berger et Joël parlaient à voix basse, pour ne pas déranger les autres, et c'était comme si on était prisonniers encore en Egypte, comme si on allait partir, et que la voix effrayante allait résonner dans le ciel et dans les montagnes, et que la lumière allait briller dans le désert » (EE: 184).

Le pouvoir magique que créait la lecture des passages du Livre du Commencement par le rabbin Joël était, à la fois, mystérieux et fascinant pour Esther : 
[...] quand il commençait, c'était comme une voix intérieure qui disait ce qu'on entendait. Il parlait de la loi et de la religion, comme si c'étaient les choses les plus faciles du monde... Il parlait du soleil, de la lune, c'étaient des contes. On ne pensait plus à l'ombre de la salle, au temps qui faisait tourner les fenêtres sur le sol. (EE:188-189).

Cependant, la conversation sur le retour à Jérusalem pour Jacques le Berger est souvent signalée par la violence et l'acharnement (peut-être même religieux). D'ailleurs, Esther remarque cet aspect violent chez lui, quand ce dernier répond à sa question sur l'existence de la Terre promise. «Le Berger m'a regardée avec violence. Son visage doux était tiré par la colère [...] Ses yeux brillaient avec impatience [...]. Lui aussi voulait voir le pays où était née la religion, où avait été écrit le premier livre » $(E E: 185)$. Ainsi, on le comprend bien lorsque Reb Joël insiste sur l'idée que la religion est la seule lumière dans la vie et que tout le reste n'est qu'ombre. Il disait que « la religion est la lumière, la seule lumière, et que toute la vie des hommes, leurs actes, tout ce qu'ils construisaient de grand et de magnifique n'étaient que des ombres » $(E E: 184)$.

De l'autre côté de l'histoire, Nejma relate elle aussi son témoignage. Une jeune Palestinienne qui, à cause de la création de l'état d'Israël, est forcée de quitter son pays et de devenir refugiée avec les autres Palestiniens dans le camp de Nour Shams. Nejma nous précise que : «Ceci est la mémoire des jours que nous avons vécus au camp de Nour Chams, telle que j'ai décidé de l'écrire, moi, Nejma, en souvenir de Saadi Abou Talib, le Beddawi, et de notre tante Aamma Houriya. En souvenir aussi de ma mère, Fatma, que je n'ai pas connue, et de mon père Ahmad» $(E E: 223)$.

Comme nous pouvons le constater, si Nejma fait ce récit, une des raisons essentielles est de démarquer à jamais les moments de souffrance et d'humiliation qu'elle et les autres réfugiés palestiniens ont vécus dans les camps de réfugiés. «Mon mari [...] m'avait demandé d'écrire tout ce que nous endurons ici, au camp de Nour Chams, afin que cela se sache, et que nul n'ose l'oublier » (EE: 234). Autrement dit, son récit est destiné à toutes les générations futures, pour que ces dernières connaissent l'injustice qu'elle a subie et pour que l'image douloureuse de l'exode palestinien soit gravée au fond de leur mémoire. On voit alors la pauvreté et la famine, des gens qui meurent en n'attendant qu'une seule ressource pour rester en vie : les camions de ravitaillement des Nations Unies qui se font de plus en plus rares au point que les déplacés se sentent complètement oubliés. "Les Nations Unies nous abandonnent, ils ne vont plus nous donner de nourriture, ni de médicaments, et nous allons tous mourir » (EE: 225). Mais l'abandon n'est pas limité au manque de nourriture et de médicaments, ce peut être beaucoup plus grave. En effet, on tourne le dos à ces réfugiés qui pensent pouvoir revenir un jour à leur vie d'antan, avant l'arrivée des camions ; ces réfugiés qui croient à la possibilité de revivre comme dans le passé :

Quand nous sommes arrivés dans le camion bâché des Nations Unies, nous ne savions pas que cet endroit allait être notre nouvelle vie. Nous pensions tous que c'était pour un jour ou deux, avant de reprendre la route. Le temps que cessent les bombardements et les combats dans les villes, et alors les étrangers nous donneraient à chacun une terre, un jardin à cultiver, une maison où on pourrait recommencer à vivre comme avant. $(E E: 226)$. 
Malheureusement, la déception fait rapidement s'écrouler les espoirs de ces réfugiés qui se sont sentis trahis. Un sentiment de conspiration ayant pour but de faire disparaître ces gens est fortement ressenti, un complot pour dépeupler les terres de ses propres habitants et y en enraciner d'autres qui arrivent de partout dans le monde. Ainsi, Nejma nous précise :

« Les soldats sont venus, ils se sont installés dans notre maison, et moi je suis montée dans un camion » (EE:229), comme si le fait de les chasser et de les faire disparaître de leur terre n'était pas suffisant, ils veulent aussi les biffer comme on le ferait d'un tout petit mot sans importance sur du papier. «Ainsi, en ont décidé les étrangers, pour que nous disparaissions à jamais de la surface de la terre » (EE : 225). Tout le monde devait être atteint par les flammes de cette décision prise. Ainsi, tous les anneaux de la chaîne devaient être étirés, on commençait d'abord par les gens les plus pauvres, ensuite on passait aux plus aisés « Dans le camion arrivaient les réfugiés, hommes, femmes, enfants au visage défait. Ce n'étaient plus les paysans pauvres, comme au début. C'étaient les gens les plus riches de Haïfa, de Jaffa, des commerçants, des avocats, ...» $(E E: 263)$.

Comme on peut le constater, le récit de Nejma est plein d'amertume et de souffrance. On peut sentir la cruauté des conditions dans lesquelles vivent ces civils impuissants: la rareté de l'eau essentielle pour boire et pour se laver, leur humiliation pour conserver l'un des droits primordiaux, celui de rester en vie. « Ils ont honte d'être devenus ce qu'ils sont, pareils à des mendiants qui quémandent leur nourriture aux portes des villes » $(E E: 263)$. Le fantôme de la mort est présent partout dans le camp, Nejma le voit se précipiter pour emporter les derniers signes de vie des autres : " J'avais su que la mort était entrée dans notre camp. Maintenant, elle allait prendre les autres animaux, et les hommes, les femmes, les enfants, l'un après l'autre » $(E E: 263)$.

D'ailleurs, tout au long de ce que Nejma raconte, la mort est l'aspect le plus marquant de son expérience. Elle assiste à la fin tragique de plusieurs de ceux qui l'entourent, elle les voit partir l'un après l'autre. La rage emporte tout, en commençant par les plus âgés et en s'attaquant même aux animaux. " Un jour, sur le chemin, j'ai vu pour la première fois une bête mourir de soif. C'était la chienne blanche de Saïd» $(E E: 232)$. Plus loin, elle explique que : « La chienne blanche était morte vraiment, j'avais vu la terreur sans but de son regard, ses yeux vitreux, j'avais entendu l'effort de son souffle qui ne voulait pas cesser, j'avais senti sous ma main le frisson très long et douloureux, puis le froid silence de son corps » (EE : 233).

C'est par ces mêmes précisions affligées que l'on peut aussi imaginer la fin de ceux qui vivent dans le camp de Nour Chams. La description donnée pour incarner les efforts funestes de l'animal mourant et essayant de s'accrocher à la vie, marque avec beaucoup d'exactitude la fatalité de cette guerre, « la terreur sans but de son regard » $(E E: 233)$. Petit à petit, la lumière de tout espoir possible s'éteint, car ceux qui y demeurent se rendent compte que c'est dans ce camp qu'ils verront leur fin. On n'a plus l'ambition de vivre, on n'a plus le droit de rêver de demain. Tous les jours se ressemblent, ils sont absolument vides et sans aucune finalité. « Le camp de Nour Chams est sans doute la fin de la terre, parce qu'il me semble qu'audelà, il ne peut rien y avoir, qu'on ne peut plus rien espérer » $(E E: 229)$, jusqu'au 
jour où naît Loula, la fille de Roumiya. C'est par les yeux innocents de cette belle créature que tout le camp voit une lueur d'espoir pour sortir de ce sombre tunnel «malgré le manque de nourriture et d'eau, il y avait un nouvel espoir pour nous [...] Il y avait un cœur dans ce camp, il y avait un centre » $(E E: 270)$.

\section{Conclusion}

Nous avons essayé de présenter quelques aspects primordiaux reflétés à travers le témoignage d'Esther et de Nejma, à savoir: la souffrance, l'humiliation, l'insécurité, la trahison d'une illusion qui pourrait submerger les esprits des rêveurs.

Par l'analyse de ces aspects, nous avons vu l'importance du « Témoignage » et son rôle multidimensionnel dans l'œuvre littéraire. En effet, en empruntant les paroles de ses personnages, l'œuvre dite «Témoignage » nous permet de nous identifier aux personnages, elle nous engage à voir, de près, des situations concrètes que ses personnages ont vécues. Elle provoque en nous des sentiments et incite notre réflexion pour réussir à comprendre l'interprétation qu'elle nous offre des faits. Cette interprétation nous porterait un message historique, idéologique, social, politique, humain ou autre.

Étoile Errante est peut-être l'histoire de deux jeunes filles ou peut-être celle de tous ceux qui ont vécu et qui continuent à vivre cette même crise ou une autre semblable. Quoi qu'il en soit, ce qui nous semble intéressant est que les deux héroïnes-témoins, Esther et Nejma, n'arrêtaient pas de penser l'une à l'autre, bien qu'elles ne se soient rencontrées qu'une seule fois en échangeant leurs noms uniquement par écrit, comme pour échapper à la haine et à la violence qui régnaient partout.

Chacune d'elle a essayé de partager sa souffrance, de libérer sa voix et de dénoncer la guerre et ses terribles conséquences. Leur témoignage porte un message évocateur pour signaler la nécessité de respecter l'humanité et de rendre hommage à la grâce du Dieu qui nous a créé tous frères et sœurs pour partager les biens de la Terre. "Nous échangerions nos cahiers pour abolir le temps, pour éteindre les souffrances et la brûlure des morts » $(E E: 316)$. Comme pour affirmer que « la Terre est à tout le monde. C'est notre mère à tous; elle est là pour le bien de tous, pour le profit de chacun, et personne n'a le droit de dire que la terre lui appartient. Elle lui est prêtée, comme la vie, d'ailleurs, comme les éléments, comme l'air » (Ezine, 1995 : 58-59). Le message est clair : la Terre est pour tous et c'est à chacun de vivre sa vie et de pratiquer ses cultes tout en respectant autrui. D'ailleurs, ce même message a été lancé par Shimon Peres, un ancien ministre israélien des Affaires Etrangères et l'un des artisans des accords d'Oslo, qui prônait souvent l'idée que si les conflits politiques peuvent se régler par des compromis, les conflits religieux ne peuvent être surmontés que par la coexistence (Lefort, $2000: 43$ ).

Or, à l'heure actuelle assombrie par une multitude de guerres et de conflits, il devient évident que nous avons de plus en plus besoin d'esprits tolérants aptes à 
accepter tout un chacun et à privilégier une coexistence humaine régie par la paix et le respect.

\section{BIBLIOGRAPHIE}

Ben JeLloun Tahar (1988), Le Clézio et Goytisolo : la question palestinienne, Le Monde des Livres, 31-32.

BURGELIN Claude (1995), Le temps des témoins, Les Cahiers de la Villa Gillet, vol. III, 79-89.

BUSNEL François (novembre 2008), J'écris pour essayer de savoir qui je suis : Le Clézio, lauréat du Prix Nobel, Lire 370, 46-49.

CORTANZE DE Gérard (2009), J.-M. G. Le Clézio: Une littérature de l'envahissement, Le Bulletin de l'Académie Royale de Langue et de Littérature Françaises de Belgique, 149-156.

EzINE Jean-Louis (1995), Ailleurs: Entretiens avec J.-M. G. Le Clézio, Paris, ARLÉA.

FElman Shoshana, and Dori LAUB (1992), Testemony: Crisis of witnessing in literature, Psychoanalysis, and History, New York, Routledge.

FLOREY Sonya (2013), L'engagement littéraire à l'ère néolibérale, Villeneuve d'Ascq, Presses universitaires du Septentrion (Col. Perspectives).

PEJOSKA-BOUCHEREAU Frosa (2014), Littérature et génocide: l'écriture testimoniale des enfants . Disponible sur http://journals.openedition.org/yod/1965 ; DOI : 10.4000/yod.1965.

ISRAELI Raphaël (2002), Jerusalem divided: The Armistice Regime 1947-1949, London, FRANK CASS \& CO. LTD.

LE CLÉZIO J.-M. G. (1992), Étoile Errante, Paris, Gallimard (édition Folio).

LE ClÉZIO J.-M. G. (2008), Dans la forêt des paradoxes, discours de réception du prix Nobel, L'Express.

Disponible sur https://www.lexpress.fr/culture/livre/dans-la-foret-des-

paradoxes-le-discours-du-nobel-j-m-g-le-clezio_720077.html.

LEFORT René (2000), Le mont du temple ou le Haram : aux sources des passions, Courrier de L'UNESCO, 40-43.

LEVY Karen D. (1998), Intersected Pasts and Problematic Futures: Oedipal Conflicts and Legendary Catastrophe in J.-M. G. Le Clézio's Onitsha and Étoile errante, International Fiction Review, 25 (1-2), 36-49.

MBASSI ATEBA Raymond (2008), Identité et fluidité dans l'œuvre de Jean-Marie Gustave Le Clézio, Une poétique de la mondialité, Paris, L'Harmattan.

SARTRE Jean-Paul (1948), Situations II, Qu'est-ce que la littérature? Paris, Gallimard.

ThiBAult Bruno (2009a), J.-M. G. Le Clézio et la métaphore exotique, Amsterdam, Rodopi.

ThiBAUlt Bruno (2009b), L’Écriture de la guerre dans « Hanné » et « Camp de Nour Shams » de J.-M. G. Le Clézio, Nouvelles Études Francophones, 24 (2), 98-107.

YANA Pierre (Octobre-Decembre1986), Écrivains dans la guerre, Revue des Sciences Humaines, 204. 